\title{
Um modelo conceitual para adaptação contínua de elementos de gamificação em ambientes educacionais
}

\author{
Vinícius Lopes ${ }^{1}$, Roseclea Duarte Medina ${ }^{1}$, \\ Giliane Bernardi $^{1}$, Felipe Becker Nunes ${ }^{2}$ \\ ${ }^{1}$ Universidade Federal de Santa Maria (UFSM) - Av. Roraima n ${ }^{\mathrm{o}} 1000$ \\ Caixa Postal 5082 - CEP 97105-900 - Santa Maria - RS - Brazil \\ ${ }^{2}$ Antonio Meneghetti Faculdade (AMF) \\ Est. Recanto Maestro n ${ }^{0} 338$ - CEP 97200-000 - Restica Sêca - RS - Brasil \\ \{vlopes, rose, giliane\}@inf.ufsm.br, nunesfb@gmail.com
}

\begin{abstract}
This paper presents a conceptual model for continuous adaptation of gamification elements based on user interactions with a gamified environment. This model considers possible changes in student's motivations throughout the use of this environment, continually personalizing its gamification elements according to these motivations. It is currently in process of analysis and refinement, for its application in a prototype of a gamified educational environment. The goal with this model is to assist developers in the construction of customized gamification environments in educational contexts.
\end{abstract}

Resumo. Neste artigo é apresentado um modelo conceitual para adaptação contínua de elementos de gamificação baseado nas interações do usuário com um ambiente gamificado. $O$ modelo considera possiveis mudanças nas motivações de um estudante durante o uso do ambiente, personalizando continuamente seus elementos de acordo com essas motivações. O mesmo encontrase, atualmente, em processo de análise e refinamento para aplicação em um protótipo de ambiente educacional gamificado e almeja-se, como possivel contribuição, que este possa auxiliar desenvolvedores na construção de ambientes gamificados personalizados em contextos educacionais.

\section{Introdução}

Tecnologias e mídias digitais estão cada vez mais presentes no cotidiano das pessoas. Por outro lado, estudantes, em ambientes educacionais, demonstram-se cada vez menos interessados nos métodos considerados "tradicionais" e passivos de ensino [Filho et al. 2018]. Desses dois fenômenos, surge a oportunidade de utilizar recursos provenientes das tecnologias digitais para promover novas estratégias de apoio aos processos de ensinoaprendizagem. Sabe-se que os jogos digitais são excelentes motivadores intrínsecos, capazes de promover a resolução de problemas e potencializar o processo de aprendizagem [Fardo 2013]. A partir disso, uma estratégia vem sendo fortemente adotada nos últimos anos em ambientes educacionais: a gamificação.

A gamificação consiste na utilização de elementos de jogos (como pontuações, missões e medalhas) em ambientes externos a um jogo [Werbach and Hunter 2012]. Sua aplicação é ampla, estando presente em espaços corporativos, educacionais, assim como em diversas outras áreas, como saúde, finanças e sustentabilidade [Deterding et al. 2011]. 
VIII Congresso Brasileiro de Informática na Educação (CBIE 2019)

Anais do XXX Simpósio Brasileiro de Informática na Educação (SBIE 2019)

No contexto educacional, o uso de ambientes gamificados tem se provado mais eficaz no processo de motivação e engajamento dos estudantes em relação a ambientes não gamificados [Oliveira et al. 2018].

Usuários de ambientes gamificados costumam ser motivados por diferentes estratégias e elementos de gamificação. Porém, boa parte dos sistemas gamificados adotam uma abordagem "one-size-fits-all" (um tamanho para todos, em tradução livre), que não considera os aspectos individuais do usuário em seu processo de gamificação [Orji et al. 2018]. Recentemente, a ideia de personalização de ambientes gamificados vem sendo adotada em diversos trabalhos, como mostra [Tondello et al. 2017b], onde fatores de personalização comuns são idade, gênero, personalidade, cultura e perfil do jogador. Dentre esses fatores, o mais utilizado é o perfil do jogador, onde o usuário de um jogo ou ambiente gamificado é classificado com base em um conjunto de características e estereótipos predominantes [Flores et al. 2016]. Existem, atualmente, diversos modelos de perfil de jogador na literatura, que classificam o usuário considerando seu comportamento, motivações e preferências, como o BrainHex de [Nacke et al. 2014] e o Hexad de [Marczewski 2015]. A maior parte desses modelos realiza esse processo de classificação de forma qualitativa, por meio de questionários.

Nos sistemas gamificados encontrados na literatura, que se utilizam de perfis de jogador como fator de personalização, esse processo é feito apenas no início, não sendo encontrados indícios da existência de sistemas que realizem uma automatização desse processo durante seu uso continuado [Toda et al. 2018]. Partindo do princípio de que um usuário pode entrar em um sistema com diferentes motivações e que as mesmas podem mudar ao longo do tempo, a personalização feita apenas em um primeiro acesso pode ser superficial ou ineficiente [Orji et al. 2018]. Surge, com isso, a necessidade de desenvolver uma solução de automatização contínua do processo de personalização para ambientes gamificados. Com base na contextualização apresentada, neste artigo é proposto um modelo conceitual para automatização de elementos de gamificação baseado no perfil de jogador e nas interações do usuário em um ambiente gamificado.

\section{Gamificação na Educação}

O uso de jogos digitais no contexto educacional proporciona ao estudante, além do conhecimento adquirido, habilidades para resolver problemas, se comunicar e colaborar com os outros [Dicheva et al. 2015]. Porém, jogos educacionais completos demandam tempo e recursos financeiros consideráveis para serem desenvolvidos, além de geralmente se focarem em objetivos específicos de aprendizagem [Kapp 2012]. Como alternativa, a gamificação emerge como uma abordagem mais simplificada, usando elementos de lógica e design de jogos para promover motivação e engajamento dos usuários.

Gamificação é definida, por [Deterding et al. 2011], como o uso de elementos de jogos em contextos externos aos de um jogo. Sua aplicação surgiu em ambientes corporativos, com sistemas de fidelização, pontuação e recompensas. Há desdobramentos do uso da gamificação em áreas de marketing, saúde, conscientização ecológica, dentre outras. No âmbito educacional, a gamificação é vista como uma tendência emergente e em constante desenvolvimento [Dicheva et al. 2015].

Diversos estudos são feitos acerca da qualidade da gamificação em ambientes educacionais [Klock et al. 2018a]. Sabe-se que, para que a gamificação 
VIII Congresso Brasileiro de Informática na Educação (CBIE 2019)

Anais do XXX Simpósio Brasileiro de Informática na Educação (SBIE 2019)

seja eficiente no processo de motivação e engajamento de estudantes, os elementos de gamificação devem ser adaptáveis e personalizáveis às necessidades e preferências do usuário [Klock et al. 2015]. Elementos de gamificação são definidos por [Werbach and Hunter 2012] como características específicas de jogos que podem ser aplicadas na gamificação, tais como pontos, recompensas, missões, placares de líderes, dentre outros. A personalização desses elementos aos diferentes tipos de usuários vem sendo aplicada em ambientes gamificados, de acordo com a literatura, considerando diferentes aspectos, tais como idade, gênero, personalidade, motivação e perfil de jogador, sendo esse último o fator mais adotado nesse processo [Klock et al. 2018b]. Este aspecto é destacado na próxima seção.

\subsection{Perfis de Jogador}

Perfis de jogador são classificações do usuário com base em seu comportamento, motivações e preferências em jogos [Klock et al. 2016]. O primeiro modelo de tipos de jogadores foi proposto por Richard Bartle, em 1996, onde jogadores de MUDs (Multi-User Dungeons, jogos online desenvolvidos, predominantemente, em forma de texto) eram classificados em relação a dois eixos: ação/interação e jogador/mundo. Tal classificação estabelece quatro tipos de jogador: conquistadores, socializadores, exploradores e assassinos [Bartle 1996]. Apesar de Bartle ser um dos pioneiros na classificação de jogadores, seu modelo é orientado para o estilo de jogo do qual ele foi originado, dificultando o processo de adaptação para outros contextos. Outros modelos, como os de [Yee et al. 2011] e [Nacke et al. 2014], classificam os jogadores conforme sua personalidade e motivações em jogos, respectivamente.

Por fim, destaca-se o modelo proposto por [Marczewski 2015], que se diferencia dos demais analisados por descrever tipos de jogador conforme suas motivações em ambientes gamificados, ao invés de jogos. Este modelo, denominado Hexad, classifica os jogadores em seis tipos: (1) Conquistador (Achiever), motivado pelo domínio, que gosta de superar desafios; (2) Socializador (Socialiser), motivado pelos relacionamentos, que gosta de interagir com outros jogadores; (3) Filantropo (Philantropist), motivado pelo propósito, altruísta que gosta de ajudar os outros de forma intrínseca; (4) Espírito Livre (Free Spirit), motivado pela autonomia e auto-expressão, gosta da liberdade para explorar um ambiente e criar coisas novas a partir dele; (5) Disruptor (Disrutptor), motivado pela mudança, gosta de contrariar as regras para observar as consequências; e, por fim (6) Jogador (Player), motivado por recompensas extrínsecas, que executa ações e tarefas para receber algo em troca.

O modelo Hexad conta com um questionário para avaliação, pontuação e classificação dos perfis predominantes [Tondello et al. 2016]. Esse questionário já foi testado e traduzido para outras línguas, dentre elas o português [Tondello et al. 2019], sendo que sua aplicação tem sido o principal método de classificação de perfis de jogador em ambientes gamificados [Toda et al. 2018]. Em [Tondello et al. 2017a] é apresentado um mapeamento de elementos de gamificação e sua relação de afinidade com cada um dos perfis do modelo Hexad, possibilitando a criação de ambientes educacionais gamificados personalizados de acordo com o estilo de cada estudante/jogador. Para além disso, considera-se que esta relação poderia servir como base para o desenvolvimento de um modelo de classificação de jogadores baseados, também, nas suas interações com esses elementos. 
VIII Congresso Brasileiro de Informática na Educação (CBIE 2019)

Anais do XXX Simpósio Brasileiro de Informática na Educação (SBIE 2019)

\section{Trabalhos Relacionados}

Alguns trabalhos relacionados a esta pesquisa foram encontrados. Dentre eles, destacase o trabalho de [Paiva et al. 2015], que apresenta uma abordagem para classificação de usuários de acordo com suas interações com um sistema gamificado para recomendar tarefas e missões. O objetivo dessa abordagem é auxiliar o professor na criação de tarefas e missões personalizadas, a fim de motivar diferentes perfis de estudantes durante o uso de sistemas gamificados. A classificação de perfis adotada neste trabalho é a de [Bartle 1996], seguindo o modelo de Processo de Recomendação Pedagógica proposto em [Paiva et al. 2013]. O trabalho não aborda nenhuma adaptação dos elementos de gamificação do sistema, se limitando apenas a recomendação do uso desses elementos baseado no perfil do usuário.

O trabalho de [Klock 2017] apresenta o desenvolvimento de um framework para o planejamento, implementação e avaliação da gamificação centrada no usuário. O framework, intitulado $5 \mathrm{~W} 2 \mathrm{H}$, é composto por sete dimensões que consideram diferentes aspectos envolvidos nesses processos, buscando estimular a interação, comunicação e desempenho dos usuários em um ambiente gamificado. Todavia, não aborda aspectos de adaptação ao perfil de jogador com base nas interações do usuário durante o uso do sistema, sendo necessária a repetição do ciclo de planejamento, implementação e avaliação para que alguma adaptação seja feita a esse sistema.

No trabalho de [Andrade 2018] são desenvolvidos dois modelos de gamificação personalizada, utilizando a classificação de [Yee et al. 2011] como base. O primeiro modelo, de Macro-gamificação, se relaciona com aspectos referentes a personalidade do usuário e a teoria de autodeterminação. O segundo modelo, de Micro-gamificação, relaciona elementos de gamificação com aspectos motivacionais. Neste estudo é feita uma comparação entre esses dois modelos de personalização e um modelo padrão, com todos os elementos disponíveis, a fim de estabelecer qual deles proporcionaria maior engajamento por parte dos estudantes. A instanciação desses modelos é aplicada após o primeiro acesso do estudante no sistema, onde o mesmo efetua um cadastro e responde a um questionário, não havendo nenhum relato de personalização durante o seu uso continuado.

De todos os trabalhos analisados durante a pesquisa, nenhum deles relatou algum processo de adaptação ou personalização de sistemas gamificados durante o uso continuado por parte dos estudantes. Apenas o trabalho de [Paiva et al. 2015] se aproxima dessa ideia ao oferecer um processo de recomendação pedagógica de elementos de gamificação baseado nas interações do usuário com o sistema. Como principal diferencial para esta pesquisa, busca-se desenvolver um modelo não apenas de recomendação, mas também de adaptação e personalização desses elementos, de forma contínua, durante o uso do sistema pelo usuário.

\section{Metodologia}

Para compreender como a gamificação vem sendo aplicada de forma adaptativa e personalizada em contextos educacionais, foi realizada uma revisão sistemática de literatura a fim de descobrir quais estratégias de adaptação vem sendo aplicadas em ambientes gamificados. Constatou-se, com essa pesquisa, que, para estabelecer um método de adaptação contínua dos elementos de gamificação em ambientes educacionais, devem ser considerados, além dos dados de contexto e a identificação do perfil de jogador, as interações do usuário durante o uso do ambiente. 
VIII Congresso Brasileiro de Informática na Educação (CBIE 2019)

Anais do XXX Simpósio Brasileiro de Informática na Educação (SBIE 2019)

Em seguida, foi realizada uma pesquisa acerca dos modelos para tipos de jogador existentes na literatura, a fim de detectar quais eram os mais comuns e quais aspectos para a definição desses tipos eram considerados. Por fim, o modelo Hexad, de [Marczewski 2015], foi a classificação escolhida como base para o modelo de adaptações desta pesquisa. Sua escolha se justifica por ser a única a apresentar tipos de jogadores específicos para ambientes gamificados [Klock et al. 2016] e um questionário empiricamente validado [Tondello et al. 2016].

Para poder avaliar as interações com o usuário, foi realizada uma pesquisa acerca dos elementos de gamificação e sua relação com perfis de jogador. No trabalho de [Tondello et al. 2017a], são classificados 49 elementos em 8 diferentes categorias de acordo com as motivações do usuário ao usar tais elementos. São elas: imersão, progressão, customização, incentivo, risco/recompensa, socialização, altruísmo e assistência. Tais categorias se relacionam, por sua vez, com os seis tipos de jogador estabelecidos no modelo Hexad. A Figura 1 representa essa relação, detalhando a intensidade (forte, moderada ou fraca) entre cada categoria e perfil de jogador.

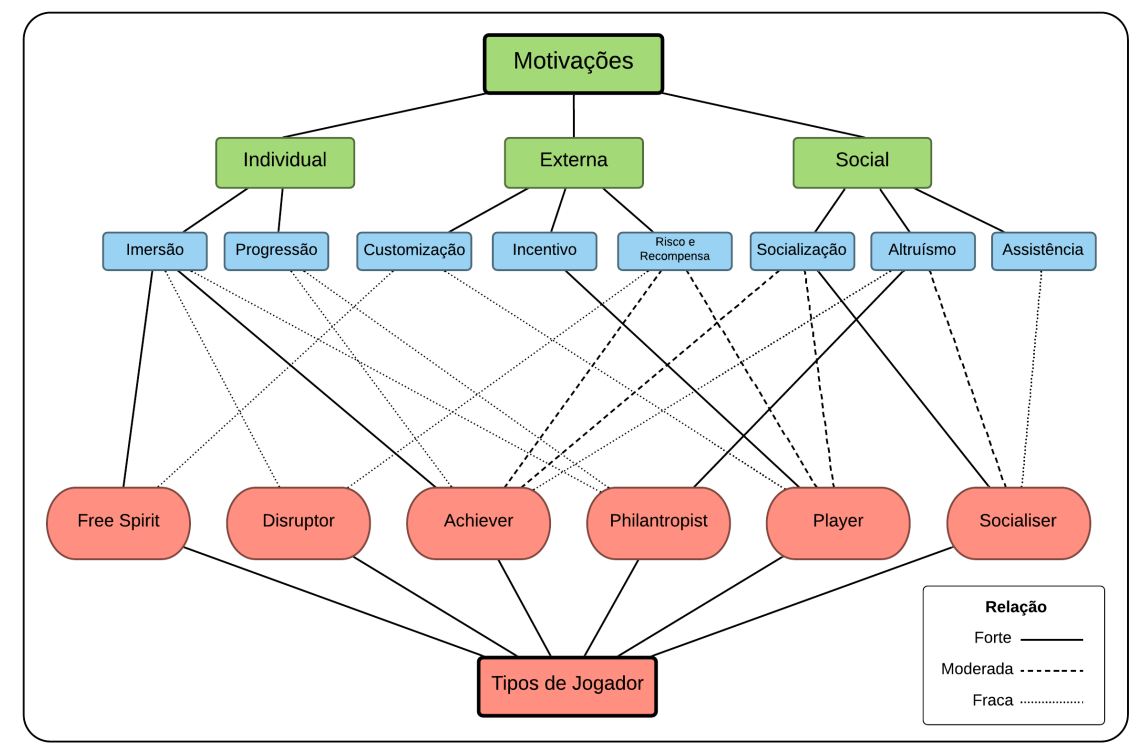

Figura 1. Relação entre motivações do usuário em ambientes gamificados e tipos de jogador. Fonte: elaborado pelo autor, adaptado de [Tondello et al. 2017a].

Com base nessas relações foi possível elaborar uma lista de possíveis interações considerando os 49 elementos de gamificação apresentados no estudo. Essa lista, elaborada com 44 possíveis interações que um usuário pode executar com elementos de gamificação, pode ser acessada em [https://bit.ly/2LBdO3u]. Por fim, com o objetivo de validar tais interações, relacionando-as com os perfis de jogador, foi desenvolvido e aplicado um questionário, destacado na seção 6.

\section{Apresentação do Modelo}

Com base nos processos metodológicos relatados, foi desenvolvido um modelo conceitual de gamificação adaptativa contínua em ambientes gamificados. A Figura 2 apresenta um fluxograma de como o modelo proposto executa os processos de adaptação de elementos de gamificação em um sistema gamificado. As etapas referentes a esses processos são explicadas a seguir. 
VIII Congresso Brasileiro de Informática na Educação (CBIE 2019)

Anais do XXX Simpósio Brasileiro de Informática na Educação (SBIE 2019)

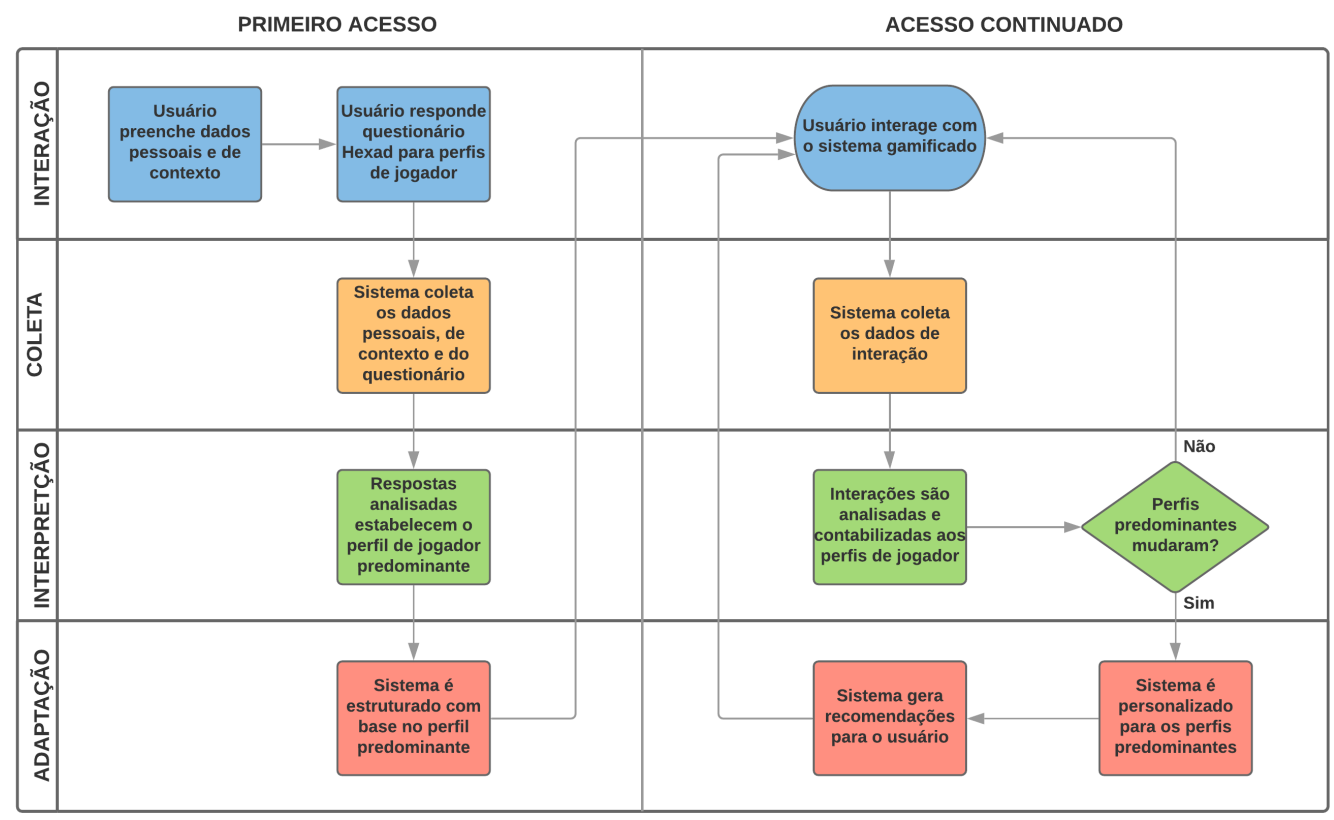

Figura 2. Fluxograma representando os processos para adaptação contínua de um sistema gamificado. Fonte: elaborado pelo autor.

Em um primeiro acesso, o usuário preenche seus dados pessoais e de contexto (como idade, gênero e escolaridade), e responde o questionário Hexad para identificação do perfil de jogador predominante. Com o conjunto dos dados pessoas, de contexto e os dados de perfil de jogador, o sistema é estruturado de forma a apresentar os elementos de gamificação que melhor representam o perfil do usuário.

Após isso, o usuário poderá interagir com todos os elementos, da forma como são apresentados pelo sistema. Cada interação possui uma relação com um ou mais perfis de jogador, e é contabilizada em forma de pontos para cada perfil com que se relaciona. Quando a pontuação de um perfil ultrapassa a de outro perfil predominante, o sistema é personalizado de forma a refletir os novos perfis predominantes. Recomendações também são geradas e passam a sugerir interações com elementos de perfis pouco predominantes, ou seja, com menor pontuação. Este processo é continuo, ou seja, se repete durante todo o período de tempo em que o usuário utiliza o sistema gamificado.

Para melhor compreensão dos processos envolvidos na adaptação, o modelo conceitual foi dividido em quatro camadas distintas: (A) interação (representando as ações de entrada da parte do usuário), (B) coleta (representando os processos de coleta de dados pelo sistema), (C) interpretação (representando os processos de análise e compreensão dos dados coletados na camada anterior) e (D) adaptação (representando os processos de adaptação feitos no sistema com base nos dados interpretados).

A camada de adaptação consiste de três diferentes etapas: estruturação, personalização e recomendação. Na etapa de estruturação, realizada apenas durante o primeiro acesso, os conteúdos e elementos de gamificação são estruturados no sistema em um layout que melhor representa o perfil de jogador predominante. Na etapa de personalização, a interface do sistema se adapta a fim de mostrar elementos de um ou mais perfis predominantes. Embora a contabilização das interações aconteça em tempo real, o processo de personalização em si não deve ser imediato pois pode prejudicar a 
VIII Congresso Brasileiro de Informática na Educação (CBIE 2019)

Anais do XXX Simpósio Brasileiro de Informática na Educação (SBIE 2019)

navegação e experiência do usuário com o ambiente. O ideal é que a personalização ocorra sempre após um período estabelecido pelo desenvolvedor, que melhor se enquadra às necessidades do ambiente (a cada novo acesso, diariamente ou semanalmente, por exemplo). Para tal, deve-se considerar aspectos tais como tempo de uso estimado e expectativa de frequência de acesso dos usuários.

Na etapa de recomendação, o sistema oferece notificações ao usuário a respeito de elementos de gamificação pertencentes a perfis não predominantes. Tal etapa é realizada com o objetivo de sugerir ao usuário a interação com outros elementos, evitando que o mesmo fique restrito ao mesmo ciclo de elementos por falta de exploração do sistema ou comodidade. Para isso, é importante que todos os elementos de gamificação implementados no sistema estejam disponíveis o tempo todo, independente da sua apresentação e disposição no layout.

\section{Resultados Parciais}

Conforme descrito na seção 4, a fim de validar empiricamente as 44 interações desenvolvidas a partir dos elementos apresentados em [Tondello et al. 2017a], um questionário foi desenvolvido buscando relacioná-las diretamente com os perfis de jogador. Almejou-se, com isso, reafirmar as hipóteses de relacionamento estabelecidas com esses elementos, mesmo após sua abstração para interações. O questionário, de caráter anônimo, aceitou submissões por um período de 6 dias, em junho de 2019, sendo amplamente divulgado em redes sociais a fim de alcançar diferentes tipos de usuários, independente de sua área de conhecimento e experiência com jogos e ambientes gamificados.

O questionário envolveu três etapas de preenchimento: na primeira etapa foi realizada a coleta de dados demográficos (como idade, gênero, escolaridade e experiência com jogos digitais e físicos); na segunda etapa foi aplicado o questionário Hexad para identificação do perfil de jogador; e por fim, na etapa final, foi questionada a probabilidade do usuário executar determinadas interações em um ambiente gamificado, baseandose na lista de 44 possíveis interações desenvolvidas. Foram recebidas 132 respostas ao questionário, com base nas quais foi possível estabelecer uma média percentual com a probabilidade com que cada uma das interações desenvolvidas seria executada.

Os 132 respondentes foram divididos em grupos, de acordo com seu perfil de jogador predominante detectados por meio das respostas da segunda etapa (questionário Hexad). Em seguida, foi calculada a probabilidade com que os indivíduos de cada um dos grupos executariam as interações propostas, com base nas respostas da etapa 3 do questionário (Tabela 1). Após isso, os 132 respondentes foram reagrupados de acordo com sua experiência com jogos, a fim de obter também as probabilidades de cada grupo em executar as interações propostas. Tais valores são representados na Figura 3.

Tabela 1. Taxa de probabilidade de interação com categorias de elementos de gamificação pelos diferentes tipos de jogador. Fonte: autoral.

\begin{tabular}{|cc|c|c|c|c|c|c|}
\hline Motivação & Categoria & Achiever & Free Spirit & Socialiser & Disruptor & Player & Philantropist \\
\hline \multirow{3}{*}{ Externa } & Customização & $69 \%$ & $71,9 \%$ & $75,9 \%$ & $52,2 \%$ & $68 \%$ & $64,5 \%$ \\
\cline { 3 - 8 } & Incentivo & $75,1 \%$ & $74,4 \%$ & $73,75 \%$ & $40,8 \%$ & $70,2 \%$ & $72,9 \%$ \\
\cline { 3 - 8 } & Risco / Recompensa & $72,4 \%$ & $70,6 \%$ & $69,6 \%$ & $51,7 \%$ & $69 \%$ & $68,4 \%$ \\
\hline \multirow{2}{*}{ Individual } & Imersão & $70 \%$ & $76 \%$ & $68,75 \%$ & $58,3 \%$ & $72,8 \%$ & $68,7 \%$ \\
\cline { 3 - 8 } & Progressão & $72,8 \%$ & $73,7 \%$ & $70,7 \%$ & $61,1 \%$ & $69,9 \%$ & $66,3 \%$ \\
\hline \multirow{3}{*}{ Social } & Altruísmo & $52,2 \%$ & $57,9 \%$ & $58,3 \%$ & $33,3 \%$ & $50,1 \%$ & $54,2 \%$ \\
\cline { 3 - 7 } & Assistência & $40 \%$ & $53,6 \%$ & $51,1 \%$ & $6,7 \%$ & $41,7 \%$ & $45 \%$ \\
\cline { 3 - 7 } & Socialização & $51,9 \%$ & $57,4 \%$ & $60,8 \%$ & $31,3 \%$ & $52,1 \%$ & $56,1 \%$ \\
\hline
\end{tabular}


VIII Congresso Brasileiro de Informática na Educação (CBIE 2019)

Anais do XXX Simpósio Brasileiro de Informática na Educação (SBIE 2019)

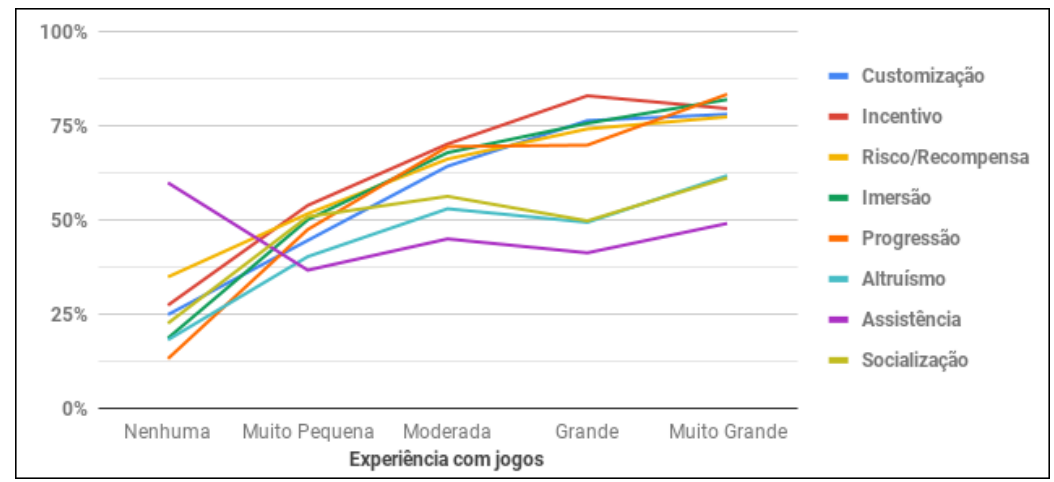

Figura 3. Relação entre os diferentes níveis de experiência do usuário com jogos e a sua probabilidade de interação com elementos de gamificação por categoria. Fonte: autoral.

Com base nestes dados foi possível constatar, por exemplo, que usuários tendem a interagir com elementos de gamificação gradativamente quanto maior a sua experiência com jogos. A única exceção para esse caso se refere aos elementos de Assistência, onde quanto menor a experiência, maior a probabilidade de um usuário interagir com esses tipos de elementos. Em relação aos perfis de jogador, alguns dados reafirmam a relação com elementos de gamificação estabelecida por [Tondello et al. 2017a]. Por exemplo, o grupo dos Socialisers apresentou uma probabilidade de 60,8\% de executar interações da categoria de Socialização, ao passo que os indivíduos do grupo dos Disruptors apresentou uma probabilidade de $31,3 \%$ de executar interações da mesma categoria.

Essas análises iniciais não consideram fatores de variação, como frequência média das interações por acesso ou o número de interações mínimas previstas de um usuário com os elementos de um sistema. Novas análises a respeito da relação entre perfis de jogador e interações com elementos de gamificação estão sendo feitas, para que, consequentemente, tais interações possam ser contabilizadas de forma correta no modelo proposto, garantindo a veracidade dos processos de adaptação de um sistema desenvolvido a partir dele.

\section{Considerações Finais}

A gamificação personalizada aplicada em ambientes educacionais ainda é um campo muito recente na literatura, com poucos estudos considerando possíveis mudanças nas motivações de um estudante durante o uso de um sistema gamificado. Neste artigo foi apresentado um modelo conceitual para automatização do processo de adaptação de elementos de gamificação baseado nas interações do usuário em um ambiente gamificado e sua relação com os perfis de jogador do modelo Hexad. A adaptação proposta pelo modelo é realizada em três etapas, sendo a primeira (estruturação) executada em um primeiro acesso e as seguintes (personalização e recomendação) ao longo do uso do sistema. O modelo conta com um conjunto de possíveis interações do usuário com um ambiente gamificado, que se encontra em processo de refinamento.

Por se tratar de um modelo conceitual em desenvolvimento, algumas decisões ainda são abordadas de forma genérica, tais como a maneira como a adaptação do sistema é feita e como as interações são interpretadas e contextualizadas pelo mesmo. Buscase, com este modelo, auxiliar desenvolvedores na construção de ambientes gamificados personalizados em contextos educacionais, sendo esta a sua maior contribuição na área da gamificação como facilitador no processo de aprendizagem. 
VIII Congresso Brasileiro de Informática na Educação (CBIE 2019)

Anais do XXX Simpósio Brasileiro de Informática na Educação (SBIE 2019)

Como trabalho subsequente, será realizado um estudo a fim de melhor compreender como os dados de contexto do usuário, em conjunto com o perfil de jogador, podem impactar na sua preferência por elementos de gamificação. Planeja-se, também, desenvolver um protótipo de sistema gamificado educacional utilizando o modelo proposto, a fim de validar o seu impacto no engajamento de estudantes com o sistema considerando suas diferentes e variantes motivações.

\section{Referências}

Andrade, F. R. H. (2018). Gamificação personalizada baseada no perfil do jogador. $\mathrm{PhD}$ thesis, Universidade de São Paulo.

Bartle, R. (1996). Hearts, clubs, diamonds, spades: Players who suit muds. Journal of MUD research, 1(1):19.

Deterding, S., Dixon, D., Khaled, R., and Nacke, L. (2011). From game design elements to gamefulness: Defining "gamification". In Proceedings of the 15th International Academic MindTrek Conference, pages 9-15, NY, USA.

Dicheva, D., Dichev, C., Agre, G., Angelova, G., et al. (2015). Gamification in education: A systematic mapping study. Educational Technology \& Society, 18(3):75-88.

Fardo, M. L. (2013). A gamificação aplicada em ambientes de aprendizagem. RENOTE - Novas Tecnologias na Educação, 11(1).

Filho, R. G. S., Silva, H. d. A., and Inocêncio, A. C. (2018). Um mapeamento sistemático sobre fatores que podem influenciar na eficiência da gamificação. In Simpósio Brasileiro de Informática na Educação-SBIE, volume 29, page 506.

Flores, T. H., Klock, A. C. T., and Gasparini, I. (2016). Identificação dos tipos de jogadores para a gamificação de um ambiente virtual de aprendizagem. RENOTE - Novas Tecnologias na Educação, 14(1).

Kapp, K. (2012). The Gamification of Learning and Instruction: Game-based Methods and Strategies for Training and Education. Pfeiffer essential resources for training and HR professionals. Wiley.

Klock, A. C. T. (2017). Análise da influência da gamificação na interação, na comunicação e no desempenho dos estudantes em um sistema de hipermídia adaptativo educacional. PhD thesis, Dissertação (Mestrado em Computação Aplicada), Universidade do Estado de Santa Catarina.

Klock, A. C. T., Flores, T. H., Gasparini, I., and HOUNSELL, M. d. S. (2016). Classificação de jogadores: um mapeamento sistemático da literatura. Anais do Simpósio Brasileiro de Jogos e Entretenimento Digital. Porto Alegre: SBC.

Klock, A. C. T., Gasparini, I., Kemczinski, A., Hounsell, M., and Isotani, S. (2015). One man's trash is another man's treasure: um mapeamento sistemático sobre as características individuais na gamificação de ambientes virtuais de aprendizagem. In Simpósio Brasileiro de Informática na Educação-SBIE, volume 26, page 539.

Klock, A. C. T., Ogawa, A. N., Gasparini, I., and Pimenta, M. S. (2018a). Does gamification matter?: A systematic mapping about the evaluation of gamification in educational environments. In Proceedings of the 33rd Annual ACM Symposium on Applied Computing, SAC '18, pages 2006-2012, New York, NY, USA. ACM. 
VIII Congresso Brasileiro de Informática na Educação (CBIE 2019)

Anais do XXX Simpósio Brasileiro de Informática na Educação (SBIE 2019)

Klock, A. C. T., Pimenta, M. S., and Gasparini, I. (2018b). A systematic mapping of the customization of game elements in gamified systems. Anais do Simpósio Brasileiro de Jogos e Entretenimento Digital. 2018.

Marczewski, A. (2015). Even Ninja Monkeys Like to Play: Gamification, Game Thinking and Motivational Design. CreateSpace Independent Publishing Platform.

Nacke, L. E., Bateman, C., and Mandryk, R. L. (2014). Brainhex: A neurobiological gamer typology survey. Entertainment computing, 5(1):55-62.

Oliveira, W. d. S., Toda, A., Isotani, S., and Bittencourt, I. I. (2018). Does gamified educational systems change students' learning behaviors? a case study with postgraduate students. RENOTE - Novas Tecnologias na Educação, 16(2).

Orji, R., Tondello, G. F., and Nacke, L. E. (2018). Personalizing persuasive strategies in gameful systems to gamification user types. In Proceedings of the $2018 \mathrm{CHI}$ Conference on Human Factors in Computing Systems, page 435. ACM.

Paiva, R., Bittencourt, I. I., and da Silva, A. P. (2013). Uma ferramenta para recomendação pedagógica baseada em mineração de dados educacionais. In Anais dos Workshops do Congresso Brasileiro de Informática na Educação, volume 2.

Paiva, R. O. A., Bittencourt, I. I., da Silva, A. P., Isotani, S., and Jaques, P. (2015). Improving pedagogical recommendations by classifying students according to their interactional behavior in a gamified learning environment. In Proceedings of the 30th Annual ACM Symposium on Applied Computing, pages 233-238. ACM.

Toda, A. M., dos Santos, W. O., Klock, A. C., Gasparini, I., Bittencourt, I. I., and Isotani, S. (2018). Frameworks para o planejamento da gamificação em contextos educacionais-uma revisão da literatura nacional. RENOTE - Novas Tecnologias na Educação, 16(2).

Tondello, G. F., Mora, A., Marczewski, A., and Nacke, L. E. (2019). Empirical validation of the gamification user types hexad scale in english and spanish. International Journal of Human-Computer Studies, 127:95-111.

Tondello, G. F., Mora, A., and Nacke, L. E. (2017a). Elements of gameful design emerging from user preferences. In Proceedings of the Annual Symposium on ComputerHuman Interaction in Play, pages 129-142. ACM.

Tondello, G. F., Orji, R., and Nacke, L. E. (2017b). Recommender systems for personalized gamification. In Adjunct Publication of the 25th Conference on User Modeling, Adaptation and Personalization, UMAP '17, pages 425-430, New York, NY, USA. ACM.

Tondello, G. F., Wehbe, R. R., Diamond, L., Busch, M., Marczewski, A., and Nacke, L. E. (2016). The gamification user types hexad scale. In Proceedings of the 2016 annual symposium on computer-human interaction in play, pages 229-243. ACM.

Werbach, K. and Hunter, D. (2012). For the win: How game thinking can revolutionize your business. Wharton Digital Press.

Yee, N., Ducheneaut, N., Nelson, L., and Likarish, P. (2011). Introverted elves \& conscientious gnomes: the expression of personality in world of warcraft. In Proceedings of the SIGCHI Conference on Human Factors in Computing Systems, pages 753-762. 